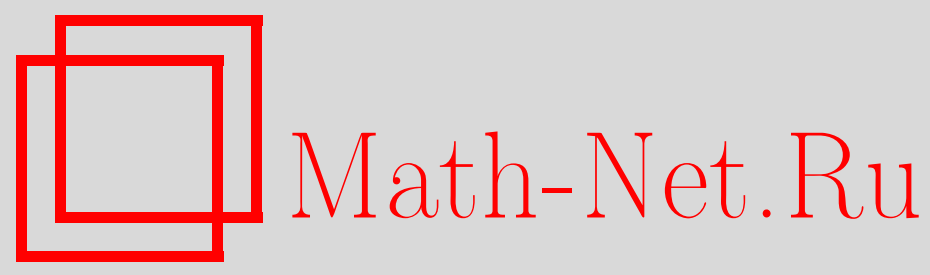

Б. А. Кац, О некоторых обобщениях понятия длины, $M a$ тем. заметки, 2001, том 70, выпуск 6, 875-881

DOI: https://doi.org/10.4213/mzm799

Использование Общероссийского математического портала Math-Net.Ru подразумевает, что вы прочитали и согласны с пользовательским соглашением http://www .mathnet.ru/rus/agreement

Параметры загрузки:

IP : 54.81 .137 .203

26 апреля 2023 г., 15:49:11

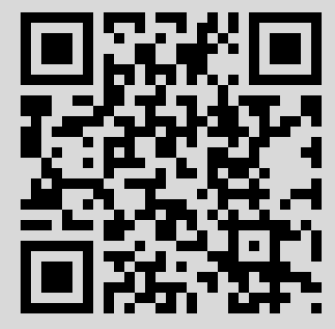




\section{О НЕКОТОРЫХ ОБОБЩЕНИЯХ ПОНЯТИЯ ДЛИНЫ}

Б. А. Кац

Для неспрямляемой дуги $\gamma \subset \mathbb{C}$ вводятся две количественные характеристики, обобщающие понятие длины. Во-первых, естественным геометрическим обобщением длины является точная верхняя грань сумм $\sum \Phi\left(a_{j}\right)$, где $a_{j}$ - длины звеньев ломаной, вписаной в дугу $\gamma$, а $\Phi$ - некоторая заданная функция. Во-вторых, длина $\gamma$ есть норма функционала $f \mapsto \int_{\gamma} f d z$ в пространстве $C(\gamma)$; его нормы в иных пространствах могут рассматриваться как аналитические обобщения длины. В статье выясняется при каких условиях из геометрической обобщенной спрямляемости кривой $\gamma$ вытекает ее аналитическая обобщенная спрямляемость.

Библиографоя: 4 названия.

1. Определения и формулировки основных результатов. Пусть $\gamma$ есть простая спрямляемая ориентированная кривая на комплексной плоскости с началом в точке $a$ и концом в точке $b$. Тем самым, на этой кривой задано отношение порядка: $z \prec w$, если $z$ предшествует $w$ при обходе $\gamma$ от $a$ к $b$. Соответственно запись $z \preccurlyeq w$ означает, что точка $z$ либо предшествует точке $w$, либо совпадает с ней. Разбиениями дуги $\gamma$ будем назьвать всевозможные строго возрастающие в смысле отношения $\prec$ конечные последовательности $\tau=\left\{z_{0}, z_{1}, \ldots, z_{n}\right\}$ точек кривой $\gamma$ такие, что $z_{0}=a, z_{n}=b, n=n(\tau)$.

Далее, заданная при $x \geqslant 0$ функция $\Phi(x)$ назьвается $\phi$ - функиией, если она непрерывна, строго возрастает, $\Phi(0)=0$ и $\lim _{x \rightarrow \infty} \Phi(x)=\infty$.

ОПРЕДЕЛЕниЕ 1 . Пусть $\Phi$ есть фиксированная $\phi$-функция. Кривая $\gamma$ называется $\Phi$-спрямляемой, если конечна величина

$$
\sigma_{\Phi}(\gamma) \equiv \sup _{\tau} \sum_{j=1}^{n(\tau)} \Phi\left(\left|z_{j}-z_{j-1}\right|\right)
$$

где верхняя грань берется по множеству всех разбиений $\gamma$.

При $\Phi(x) \equiv x^{p}$ мы будем говорить о $p$-спрямляемости и писать $\sigma_{p}$ вместо $\sigma_{\Phi}$. Очевидно, 1-спрямляемые кривые спрямляемы, а с ростом $p$ класс $p$-спрямляемых кривых строго расширяется. Для любого $p>1$ можно указать $p$-спрямляемую кривую, не являющуюся $q$-спрямляемой ни при одном $q<p$. При $p<1$ класс $p$-спрямляемых кривых пуст.

Пусть теперь $\mathbb{P}$ есть множество всех алгебраических многочленов от переменной $z$, т.е. каждый элемент $\mathbb{P}$ имеет вид $f(z)=f_{0}+f_{1} z+f_{2} z^{2}+\cdots+f_{m} z^{m}$, где $f_{0}, f_{1}, \ldots, f_{m}-$ 
постоянные числа, $m=m(f)$. Обозначим через $\mathbb{P}_{0} \subset \mathbb{P}$ множество всех многочленов, обращающихся в нуль в точке $a$, а через $\mathbb{P}^{*}$ множество всех многочленов, не равных тождественно нулю. Если кривая $\gamma$ спрямляема и $f \in \mathbb{P}_{0}$, то, очевидно, $f(b)=\int_{\gamma} f^{\prime}(z) d z$ и, следовательно, $|f(b)| \leqslant \lambda\left\|f^{\prime}\right\|_{C(\gamma)}$, где $\|\cdot\|_{C(\gamma)}$ есть равномерная норма на $\gamma$, т.е.

$$
\|g\|_{C(\gamma)} \equiv \sup \{|g(z)|: z \in \gamma\}
$$

а постоянная $\lambda$ не зависит от $f$. Наилучшим значением этой постоянной является длина $\gamma$, т.е. мы можем положить здесь $\lambda=\sigma_{1}(\gamma)$. Можно показать, что величина

$$
\sup \left\{\frac{|f(b)|}{\left\|f^{\prime}\right\|_{C(\gamma)}}: f \in \mathbb{P}_{0}, f^{\prime} \in \mathbb{P}^{*}\right\}
$$

конечна тогда и только тогда, когда кривая $\gamma$ спрямляема. Заменяя здесь равномерную норму любой другой, мы получим некоторую характеристику кривой $\gamma$, которая может оказаться конечной и для некоторых неспрямляемых кривьх. Пусть $\mathfrak{n}$ есть некоторая норма, определенная на $\mathbb{P}$ и такая, что $\mathfrak{n}(\mathbf{1})=1$, где $\mathbf{1}$ означает многочлен, тождественно равный единице.

ОПРЕДЕЛЕниЕ 2. Кривая $\gamma$ называется [n]- спрямляемой, если конечна величина

$$
\lambda_{\mathfrak{n}}(\gamma) \equiv \sup \left\{\frac{|f(b)|}{\mathfrak{n}\left(f^{\prime}\right)}: f \in \mathbb{P}_{0}, f^{\prime} \in \mathbb{P}^{*}\right\}
$$

Ниже мы используем следующий класс норм. Пусть $\psi(x)$ есть фиксированная $\phi$-функция. Пространство $H_{\psi}(\gamma)$ состоит из всех непрерьвньгх на $\gamma$ функций $f(z)$, удовлетворяющих условию

$$
\left|f\left(z_{1}\right)-f\left(z_{2}\right)\right| \leqslant c \psi\left(\left|z_{1}-z_{2}\right|\right)
$$

при всех $z_{1,2} \in \gamma$ и некотором $c=c(f)>0$. Точная нижняя грань значений $c$, для которых выполняется это неравенство, обозначается ниже через $c_{\psi}(f)$; величина $\mathfrak{n}_{\psi}(f)=$ $|f(a)|+c_{\psi}(f)$ является нормой пространства $H_{\psi}(\gamma)$. Если

$$
\lim _{x \rightarrow 0} \frac{\psi(x)}{x}=0
$$

то это пространство состоит из постоянных; напротив, если величина $x / \psi(x)$ ограничена при мальх $x$, то $\mathbb{P} \subset H_{\psi}(\gamma)$. При $\psi(x) \equiv x^{\nu}, 0<\nu \leqslant 1$, мы будем говорить о $[\nu]$-спрямляемости и писать $H_{\nu}, c_{\nu}, h_{\nu}$ и $\lambda_{\nu}$ вместо $H_{\psi}, c_{\psi}, \mathfrak{n}_{\psi}$ и $\lambda_{\mathfrak{n}_{\psi}}$ соответственно. Если $0<\mu<\nu \leqslant 1$, то всякая $[\mu]$-спрямляемая кривая является $[\nu]$-спрямляемой, т.е. с ростом $\nu$ класс $[\nu]$-спрямляемых кривых расширяется. Основным результатом данной работы является

ТЕОРема 1. Пусть $\Phi$ и -логарифмически выпуклые ф-функиии такие, что $\psi(x) \geqslant x$ при мальх $x$ и сходится ряд

$$
\sum_{n=1}^{\infty} \varphi\left(\frac{1}{n}\right) \psi\left(\varphi\left(\frac{1}{n}\right)\right)<\infty
$$

где ч есть функиия, обратная к $\Phi$. Тогда всякая $\Phi$-спрямляемая кривая $\left[\mathfrak{n}_{\psi}\right]-$ спрямляема. 
СлЕДСТВИЕ 1. Если $\Phi(x)$ есть логарифмически выпуклая ф-функиия, то при условии

$$
\sum_{n=1}^{\infty} \varphi^{1+\nu}\left(\frac{1}{n}\right)<\infty
$$

всякая $\Phi$-спрямляемая кривая является $[\nu]$-спрямляемой.

В частности, всякая $p$-спрямляемая кривая $[\nu]$-спрямляема при условии $1 \geqslant \nu>$ $p-1>0$, а при $p<2$ всякая $p$-спрямляемая кривая [1]-спрямляема.

Напротив, из $[\nu]$-спрямляемости кривой, вообще говоря, не следует, что она $p$-спрямляема для какого-либо $p$. На это указьвает

ТЕОремА 2. Существует кривая $\gamma$, для которой $\sigma_{p}(\gamma)=\infty$ при любом $p \geqslant 1$, но $\lambda_{1}(\gamma)<\infty$.

2. Доказательства и комментарии. Доказательства теорем основываются на ряде лемм, первая из которых является известным результатом. Чтобы ее сформулировать, напомним определение так назьваемой $\Phi$-вариации (см. [1], а также [2]). Если $\Phi(x)$ есть фиксированная ф-функция, то $\Phi$ - вариацией функции $x(t)$, заданной на отрезке $I=[0,1]$, называют величину

$$
v_{\Phi}(x) \equiv \sup _{\tau} \sum_{j=1}^{n(\tau)} \Phi\left(\left|x\left(t_{j}\right)-x\left(t_{j-1}\right)\right|\right)
$$

где верхняя грань берется по множеству всех разбиений отрезка $I$, т.е. конечных последовательностей $\tau=\left\{t_{0}, t_{1}, \ldots, t_{n}\right\}$ точек этого отрезка таких, что $0=t_{0}<t_{1}<\cdots<$ $t_{n}=1, n=n(\tau)$. Класс $V_{\Phi}(I)$ состоит из всех заданных на $I$ функций конечной $\Phi$-вариации. Хорошо известен следуюший результат Юнга (см. [1, разд. 4]).

ЛЕмма 1. Пусть $\Phi$ и суть две логарифмически выпуклые ф-функиии, а $и ~ и$ $\psi$ - их обратные функиии. Если функиии $x(t) \in V_{\Psi}(I)$ и $y(t) \in V_{\Phi}(I)$ не имеют на $I$ общих точек разрыва и сходится ряд

$$
\sum_{n=1}^{\infty} \varphi\left(\frac{1}{n}\right) \psi\left(\frac{1}{n}\right)<\infty
$$

то интеграл Стилтьеса $\int_{0}^{1} x d y$ существует и при $x(0)=0$ допускает оценку

$$
\left|\int_{0}^{1} x d y\right| \leqslant \psi\left(v_{\Psi}(x)\right) \varphi\left(v_{\Phi}(y)\right)+\sum_{n=1}^{\infty} \psi\left(\frac{v_{\Psi}(x)}{n}\right) \varphi\left(\frac{v_{\Phi}(y)}{n}\right) .
$$

Понятие $\Phi$-вариации без труда переносится с отрезка на ориентированную кривую $\gamma$, поскольку использует лишь упорядоченность области определения функции. Обозначим

$$
v_{\Phi}(x ; \gamma) \equiv \sup _{\tau} \sum_{j=1}^{n(\tau)} \Phi\left(\left|x\left(z_{j}\right)-x\left(z_{j-1}\right)\right|\right)
$$


где верхняя грань берется по множеству всех разбиений кривой $\gamma$, а функция $x(z)$ определена на этой кривой. Аналогично, класс $V_{\Phi}(\gamma)$ состоит из всех заданных на $\gamma$ функций конечной $\Phi$-вариации. Нетрудно определить и интеграл Стилтьеса $\int_{\gamma} x d y$ по ориентированной кривой $\gamma$. Он существует и равен $A$, если для любого $\varepsilon>0$ можно указать $\delta=\delta(\varepsilon)>0$ такое, что

$$
\left|A-\sum_{j=1}^{n(\tau)} x\left(w_{j}\right)\left(y\left(z_{j}\right)-y\left(z_{j-1}\right)\right)\right| \leqslant \varepsilon
$$

для любого разбиения $\tau$ такого, что $\left|z_{j}-z_{j-1}\right| \leqslant \delta, j=1,2, \ldots, n(\tau)$, и любых точек $w_{1}, w_{2}, \ldots, w_{n}$ таких, что $a=z_{0} \preccurlyeq w_{1} \preccurlyeq z_{1} \preccurlyeq w_{2} \preccurlyeq \cdots \preccurlyeq w_{n} \preccurlyeq z_{n}=b$. Далее, пусть $\zeta=\zeta(t)$ есть непрерьвное взаимно однозначное отображение $I$ на $\gamma$, причем $\zeta(0)=a$, $\zeta(1)=b$. Очевидно, интегралы Стилтьеса $\int_{\gamma} x d y$ и $\int_{0}^{1} x(\zeta(t)) d y(\zeta(t))$ существуют или не существуют одновременно, причем в первом случае они совпадают. Поэтому из леммы 1 немедленно следует

Лемма 2. Пусть $\Phi$ и суть две логарифмически выпуклые ф-функиии, а $и ~ и$ $\psi$ - их обратные функиии. Если функиии $x(z) \in V_{\Psi}(\gamma)$ и $y(z) \in V_{\Phi}(\gamma)$ не имеют на $\gamma$ общих точек разрыва и сходится ряд (2), то интеграл Стилтьеса $\int_{\gamma} x d y$ существует и при $x(a)=0$ допускает оценку

$$
\left|\int_{\gamma} x d y\right| \leqslant \psi\left(v_{\Psi}(x ; \gamma)\right) \varphi\left(v_{\Phi}(y ; \gamma)\right)+\sum_{n=1}^{\infty} \psi\left(\frac{v_{\Psi}(x ; \gamma)}{n}\right) \varphi\left(\frac{v_{\Phi}(y ; \gamma)}{n}\right)
$$

Пусть теперь $y(z) \equiv z$. Тогда $v_{\Phi}(y ; \gamma)=\sigma_{\Phi}(\gamma)$ и результат леммы 2 приобретает следующий вид.

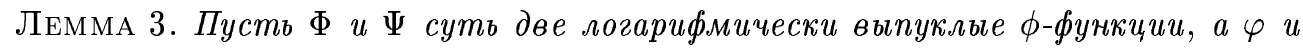
$\psi$ - их обратные функиии. Если кривая $\gamma$ является $\Phi$-спрямляемой, $x(z) \in V_{\Psi}(\gamma)$ u сходится ряд (2), то интеграл Стилтьеса $\int_{\gamma} x d z$ существует и при $x(a)=0$ допускает оиенку

$$
\left|\int_{\gamma} x(z) d z\right| \leqslant \psi\left(v_{\Psi}(x ; \gamma)\right) \varphi\left(\sigma_{\Phi}(\gamma)\right)+\sum_{n=1}^{\infty} \psi\left(\frac{v_{\Psi}(x ; \gamma)}{n}\right) \varphi\left(\frac{\sigma_{\Phi}(\gamma)}{n}\right)
$$

Теперь мы можем выяснить при каких условиях существует интеграл Стилтьеса от многочлена по неспрямляемой дуге.

ЛЕмма 4. Пусть Ф есть логарифмически выпуклая ф-функиия, кривая $\gamma$ является $\Phi$-спрямляемой $и f \in \mathbb{P}$. Тогда интеграл Стилтьеса $\int_{\gamma} f d z$ существует при условии сходимости ряда

$$
\sum_{n=1}^{\infty} \varphi^{2}\left(\frac{1}{n}\right)<\infty
$$


ДоказАтЕльство. Очевидно, $\mathbb{P} \subset H_{1}(\gamma)$, т.е. для любых точек $z_{1} \in \gamma$ и $z_{2} \in \gamma$ справедливо неравенство $\left|f\left(z_{1}\right)-f\left(z_{2}\right)\right| \leqslant h_{1}\left|z_{1}-z_{2}\right|, h_{1}=h_{1}(f)$. Положим $f^{*}(z)=f(z) / h_{1}$. Тогда $\left|f^{*}\left(z_{1}\right)-f^{*}\left(z_{2}\right)\right| \leqslant\left|z_{1}-z_{2}\right|$ и, следовательно, $v_{\Phi}\left(f^{*} ; \gamma\right) \leqslant \sigma_{\Phi}(\gamma)$. Таким образом, $f^{*} \in V_{\Phi}(\gamma)$ и в силу леммы 3 интеграл $\int_{\gamma} f^{*} d z$ существует при условии сходимости ряда (4). Но тогда, очевидно, существует и интеграл

$$
\int_{\gamma} f d z=h_{1} \int_{\gamma} f^{*} d z
$$

ЛЕмма 5. Пусть Ф есть логарифмически выпуклая ф-функиия, для которой сходится ряд (4), кривая $\gamma$ является $\Phi$-спрямляемой $и f \in \mathbb{P}$. Тогда

$$
\int_{\gamma} f^{\prime} d z=f(b)-f(a)
$$

ДокАЗАТЕЛьСтво. В силупредыдущей леммы интеграл в левой части равенства (5) существует. Очевидно, интеграл $\int_{\gamma} d f$ также существует и равен $f(b)-f(a)$. Таким образом, мы должны доказать равенство

$$
\int_{\gamma} f^{\prime} d z=\int_{\gamma} d f
$$

причем существование интегралов в обеих его частях уже установлено. Рассмотрим любое разбиение $\tau=\left\{z_{0}, z_{1}, \ldots, z_{n}\right\}$ дуги $\gamma$. Обозначим через $s_{j}$ прямолинейньй отрезок, соединяющий точки $z_{j-1}$ и $z_{j}$. Тогда одна из интегральных сумм, соответствующих левой части (6), имеет вид

$$
S_{1}=\sum_{j=1}^{n} f^{\prime}\left(z_{j-1}\right)\left(z_{j}-z_{j-1}\right)=\sum_{j=1}^{n} \int_{s_{j}} f^{\prime}\left(z_{j-1}\right) d z
$$

а интегральная сумма для правой части (6) есть

$$
S_{2}=\sum_{j=1}^{n} f\left(z_{j}\right)-f\left(z_{j}\right)=\sum_{j=1}^{n} \int_{s_{j}} f^{\prime}(z) d z
$$

Разность этих сумм допускает оценку

$$
\left|S_{1}-S_{2}\right| \leqslant \sum_{j=1}^{n} \int_{s_{j}}\left|f^{\prime}(z)-f^{\prime}\left(z_{j-1}\right)\right||d z| \leqslant h_{1}\left(f^{\prime}\right) \sum_{j=1}^{n}\left|z_{j}-z_{j-1}\right|^{2} .
$$

Очевидно, $\left|z_{j}-z_{j-1}\right|^{2}=\varphi^{2}\left(\Phi\left(\left|z_{j}-z_{j-1}\right|\right)\right)$. Поскольку последовательность чисел $\varphi^{2}(1 / n), n=1,2, \ldots$, монотонно убывает и ряд (4) сходится, то $\varphi^{2}(1 / n)=o(1 / n)$ (см., например, [3]). Поэтому функция $\rho(x) \equiv \varphi^{2}(x) / x$ стремится к 0 при $x \rightarrow 0$; тогда этим 
свойством обладает и функция $\rho^{*}(x)=\max \left\{\rho\left(x^{\prime}\right): 0<x^{\prime} \leqslant x\right\}$. Теперь мы можем продолжить оценку разности интегральных сумм:

$$
\left|S_{1}-S_{2}\right| \leqslant h_{1}\left(f^{\prime}\right) \sum_{j=1}^{n} \rho\left(\Phi\left(\left|z_{j}-z_{j-1}\right|\right) \Phi\left(\left|z_{j}-z_{j-1}\right|\right)\right) \leqslant h_{1}\left(f^{\prime}\right) \sigma_{\Phi}(\gamma) \rho^{*}(\Phi(\delta))
$$

где $\delta=\max \left\{\left|z_{j}-z_{j-1}\right|: j=1,2, \ldots, n(\tau)\right\}$. Итак, эта разность стремится к нулю вместе с $\delta$, что и завершает доказательство леммы.

Теперь мы можем закончить доказательство теоремы 1 . Пусть $f \in \mathbb{P}_{0}$ и $f^{\prime} \in \mathbb{P}^{*}$. Поскольку $\psi(x) \geqslant x$ при малых $x$, то сходимость ряда (1) влечет выполнение условия (4). Тогда согласно двум последним леммам интеграл $\int_{\gamma} f^{\prime} d z$ существует и равен $f(b)$. Как уже отмечалось, при условии ограниченности величины $x / \psi(x)$ пространство $H_{\psi}(\gamma)$ содержит все многочлены. Обозначим $f_{1}(z)=\left(f^{\prime}(z)-f^{\prime}(a)\right) / c_{\psi}\left(f^{\prime}\right)$. Очевидно,

$$
f(b)=f^{\prime}(a)(b-a)+c_{\psi}\left(f^{\prime}\right) \int_{\gamma} f_{1} d z
$$

Поскольку $\left|f_{1}\left(z^{\prime}\right)-f_{2}\left(z^{\prime \prime}\right)\right| \leqslant \psi\left(\left|z^{\prime}-z^{\prime \prime}\right|\right)$ для любых $z^{\prime} \in \gamma$ и $z^{\prime \prime} \in \gamma$, то $f_{1} \in V_{\Xi}(\gamma)$, где $\Xi(x)=\Phi(\Psi(x))$, и $v_{\Xi}\left(f_{1}\right) \leqslant \sigma_{\Phi}(\gamma)$. Обратная к $\Xi(x)$ функция есть $\xi(x)=\psi(\varphi(x))$, поэтому при вьполнении условия (1) мы согласно (3) имеем

$$
\left|\int_{\gamma} f_{1}(z) d z\right| \leqslant \psi(\varphi(\sigma)) \varphi(\sigma)+\sum_{n=1}^{\infty} \psi\left(\varphi\left(\frac{\sigma}{n}\right)\right) \varphi\left(\frac{\sigma}{n}\right)
$$

где $\sigma=\sigma_{\Phi}(\gamma)$. Обозначим правую часть $(8)$ через $Z_{\varphi, \psi}(\sigma)$. Эта величина не зависит от $f$. Теперь из (7) следует оценка

$$
|f(b)| \leqslant \mathfrak{n}_{\psi}\left(f^{\prime}\right) \max \left\{|b-a|, Z_{\varphi, \psi}\left(\sigma_{\Phi}(\gamma)\right)\right\}
$$

которая означает, что кривая $\gamma$ является $\left[\mathfrak{n}_{\psi}\right]$-спрямляемой. Теорема доказана.

Отметим, что при условиях этой теоремы $|b-a|=\varphi(\Phi(|b-a|)) \leqslant \varphi\left(\sigma_{\Phi}(\gamma)\right)$. Поэтому из доказательства теоремы 1 следует оценка

$$
\lambda_{\mathfrak{n}_{\psi}}(\gamma) \leqslant \max \left\{\varphi(\sigma), Z_{\varphi, \psi}(\sigma)\right\}, \quad \sigma=\sigma_{\Phi}(\gamma)
$$

означающая, что при фиксированных $\Phi$ и $\psi$ "длина" $\lambda_{n_{\psi}}(\gamma)$ оценивается сверху величиной, зависящей только от “длины” $\sigma_{\Phi}(\gamma)$.

Положив в теореме $1 \psi(x)=x^{\nu}$, получаем следствие 1 . Это следствие влечет, в частности, доказанное в [4] утверждение о [ $\nu]$-спрямляемости любой $p$-спрямляемой кривой при условии $\nu>p-1$. Докажем теперь теорему 2 , показывающую, что обратное утверждение неверно. Ее доказательство основано на следующем достаточном условии [1]-спрямляемости, установленном в [4]. 
ЛЕмма 6. Если кривая $\gamma$ имеет нулевую площадь и однозначная в $\mathbb{C} \backslash \gamma$ функиия

$$
K_{\gamma}(z)=\frac{1}{2 \pi i} \log \frac{z-b}{z-a}
$$

интегрируема в любой конечной части плоскости, то $\lambda_{1}(\gamma)<\infty$.

Теперь для доказательства теоремы 2 достаточно фиксировать три монотонно убывающие и стремящиеся к нулю последовательности вещественных чисел $\left\{x_{n}\right\},\left\{y_{n}\right\}$ и $\left\{\varepsilon_{n}\right\}$ такие, что $\varepsilon_{n}<x_{n}-x_{n+1}$ при $n=1,2, \ldots$, и $\sum_{n=1}^{\infty} y_{n}^{p}=\infty$ для любого $p \geqslant 1$, и рассмотреть кривую $\gamma$, состоящую из горизонтальных отрезков $\left[x_{n+1}, x_{n}-\varepsilon_{n}\right]$ и $\left[x_{n}-\varepsilon_{n}+i y_{n}, x_{n}+i y n\right]$ и вертикальных отрезков $\left[x_{n}, x_{n}+i y_{n}\right]$ и $\left[x_{n}-\varepsilon_{n}, x_{n}-\varepsilon_{n}+i y_{n}\right]$, где $n=1,2, \ldots$. Очевидно, эта кривая имеет нулевую площадь, и особенности функции $K_{\gamma}(z)$ вблизи ее концов 0 и $x_{1}$ имеют логарифмический порядок роста, т.е. эта функция интегрируема в любой конечной части плоскости в сколь угодно высокой степени. Поэтому эта кривая [1]-спрямляема в силу последней леммы. Но предположение о $p$-спрямляемости этой кривой влечет сходимость ряда $\sum y_{n}^{p}$, и поэтому эта кривая не является $p$-спрямляемой ни при каком $p \geqslant 1$.

Таким образом, обобщенная спрямляемость в смысле определения 1 влечет спрямляемость в гёльдоровой шкале в смысле определения 2 , но не эквивалентна ей. Было бы интересно описать шкалу норм, спрямляемость относительно которых эквивалентна $p$-спрямляемости.

\section{СПИСОК ЦИТИРОВАННОЙ ЛИТЕРАТУРЫ}

[1] Young L.C. General inequalities for Stieltjes integrals and the convergence of Fourier series // Math. Ann. 1938. V. 115. № 4. P. 581-612.

[2] Lesniewicz R., Orlicz W. On generalized variation. II // Studia Math. 1973. V. 45. № 1. P. 71-109.

[3] Фихтенгольц Г. М. Курс дифференциального и интегрального исчисления. Т. II. М.: Наука, 1969.

[4] Kats B. A. The inequalities for polynomials and integration over fractal arcs // Canad. Math. Bull. (to appear). 